\title{
Convergence Behavior in Line Profile Analysis Using Convolutional Multiple Whole-Profile Software
}

\author{
Masayoshi Kumagai ${ }^{1, a,{ }^{\star}}$, Tomohiro Uchida ${ }^{1}$, Kodai Murasawa ${ }^{2, b}$, \\ Masato Takamura ${ }^{3, c}$, Yoshimasa Ikeda ${ }^{3, d}$, Hiroshi Suzuki ${ }^{4, e}$, \\ Yoshie Otake $^{3, \mathrm{f}}$, Takayuki Hama ${ }^{5, \mathrm{~g}}$, Shinsuke Suzuki ${ }^{2, \mathrm{~h}}$ \\ ${ }^{1}$ Faculty of Engineering, Tokyo City University, Japan \\ ${ }^{2}$ Faculty of Science and Engineering, Waseda University, Japan \\ ${ }^{3}$ RIKEN Center for Advanced Photonics, RIKEN, Japan \\ ${ }^{4}$ Materials Sciences Research Center, Japan Atomic Energy Agency, Japan \\ ${ }^{5}$ Graduate School of Energy Science, Kyoto University, Japan \\ amkumagai@tcu.ac.jp, ${ }^{\mathrm{b}}$ murasawa@akane.waseda.jp, ${ }^{c}$ takamura@riken.jp, \\ dyoshimasa.ikeda@riken.jp, ${ }^{\mathrm{e}}$ suzuki.hiroshi07@jaea.go.jp, ${ }^{\mathrm{f}}$ yotake@riken.jp, \\ ghama@energy.kyoto-u.ac.jp, 'suzuki-s@waseda.jp
}

\section{Keywords: Neutron Diffraction, Line Profile Analysis, Microstructure, CMWP}

\begin{abstract}
The convergence behavior of the parameters related to microstructural characteristics $a-e$ was studied during optimizations in a common line profile analysis software program based on the convolutional multiple whole profile (CMWP) method. The weighted sums of squared residual (WSSR) was a criterion of the optimization. The parameters $b$ and $d$, which are related to the dislocation density and to the crystallite size, respectively, strongly affect the line profile shape. Therefore, the distributions of WSSRs on the space parameters $b$ and $d$ were first observed. The variation trajectory of parameters $b$ and $d$ during iterative calculations with several values of parameter $e$ was then observed, along with the variations when all of the parameters were variable. In the case where only three parameters were variable, we found that a smaller initial value of $e$ should be chosen to ensure stability of the calculations. In the case where all parameters were variable, although all of the results converged to similar values, they did not precisely agree. To attain accurate optimum values, a two-step procedure is recommended.
\end{abstract}

\section{Introduction}

The mechanical properties of materials depend on microstructural features such as dislocations. Therefore, the characterization of such features is important in materials and mechanical engineering. Microstructural features can be observed by several methods. One approach is Xray/neutron diffraction line profile analysis (LPA). In this method, microstructural features are characterized through inverse analysis of diffraction line profiles. LPA is a nondestructive measurement, which enables its use in in situ measurements during, for example, tensile tests. Pulsed neutron sources in large proton accelerator facilities are suitable for in situ measurements because of the high flux of the neutron beam and the time-of-flight (TOF)-type diffractometer used in these facilities. In addition, whole diffraction profiles can be obtained within a short measurement time.

Several line profile methods have been developed and reviewed in a book [1]. The modified Williamson-Hall/Warren-Averbach method [2] is one of the common methods in the 2000s. However, in this method, each diffraction peak is fitted individually even though whole profiles can be obtained via the measurements with a TOF diffractometer. For the efficient analysis of

(c) (1) Content from this work may be used under the terms of the Creative Commons Attribution 3.0 license. Any further distribution of this work must maintain attribution to the author(s) and the title of the work, journal citation and DOI. Published under license by Materials Research Forum LLC. 
measured profiles, whole-profile analysis methods [3-6] are better than that individual-peak methods, especially for TOF measurements.

The convolutional multiple whole profile (CMWP) fitting method and its software [6] is a common method. The software is convenient approach to whole-profile analysis, and users can obtain parameters related to a sample's microstructure. However, because many parameters are optimized during the CMWP fitting process, users must consider how well to optimize these parameters. Therefore, we studied the convergence behavior in LPA using CMWP software to understand the phenomenon and propose a suitable procedure.

\section{Theorem}

In the CMWP method, each measured diffraction pattern is fitted by a theoretical diffraction pattern calculated with five fitting parameters (anisotropy parameter $q$, variance of the lognormal crystallite size distribution $\sigma_{\mathrm{LN}}$, effective outer cutoff radius of dislocation $R_{\mathrm{e}}{ }^{*}$, dislocation density $\rho$, and crystallite size $L_{0}$ ) through iterative calculations. These five parameters correspond to the parameters $a, b, c, d$, and $e$ in the CMWP software as follows:

$$
\begin{aligned}
& q=a \\
& \sigma_{\mathrm{LN}}=\frac{c}{\sqrt{2}} \\
& \rho=\frac{2}{\pi\left(b_{\text {Burgers }} d\right)^{2}} \\
& R_{\mathrm{e}}^{*}=\frac{\exp \left(-\frac{1}{4}\right)}{2 e} \\
& L_{0}=\frac{2}{3} \exp \left(\frac{5}{4} c^{2}+b\right)
\end{aligned}
$$

\section{Experimental}

The measured diffraction line profile for 780MPa-grade steel [7] was used in this study. The profiles covering the scattering vector region from 3.75 to $12.5 \mathrm{~nm}^{-1}$, which includes six peaks (Fig. 1), were used. The diffraction indices of the six peaks of $\alpha \mathrm{Fe}$ in this region are 110, 200, 211, 220, 310, and 222. The other common analysis conditions are shown in Table 1. The background of the line profile was fixed throughout this study.

Initially, weighted sums of squared residuals

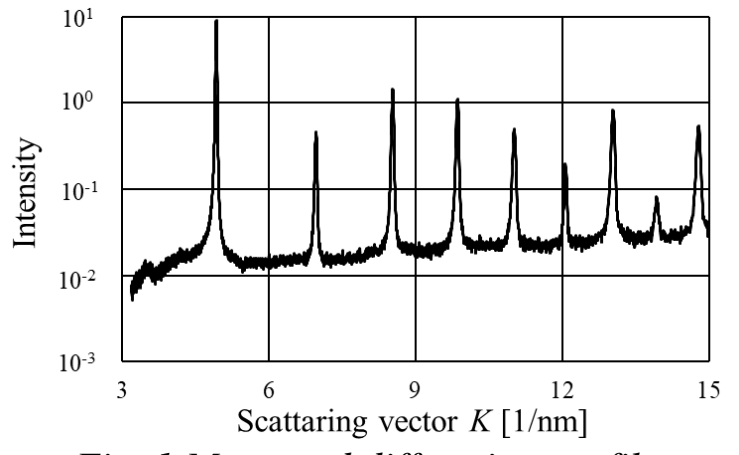

Fig. 1 Measured diffraction profile.

Table 1 Analysis conditions

\begin{tabular}{|l|c|}
\hline Latice constant [nm] & 0.28665 \\
\hline$C_{\mathrm{h} 00}$ & 0.284 \\
\hline Fit limit & $1 \times 10^{-9}$ \\
\hline Use weights & No \\
\hline Fit peak pos & Yes \\
\hline Instrum. Profiles & $\mathrm{LaB}_{6}$ \\
\hline
\end{tabular}
(WSSRs) at several values of parameters $b$ and $d$ were obtained. The other parameters $-a, c$, and $e$-were fixed as arbitrary values.

We next performed optimizations with i) three parameters $(b, d$, and $e$ ) and ii) all parameters varied with four arbitrary initial values; the trajectories of $b$ and $d$ were drawn on the contour maps of WSSRs in $b-d$ space.

\section{Results}

Figure 2 shows the WSSRs contour map at each $b$ and $d$ value. The contour colors were interpolations of calculated values shown as plots in the figure. Regions where the WSSR is smaller than in other regions (hereafter referred to as valleys) exist, similar to an inverse 
proportion in $d-b$ space. The parameters $b$ and $d$ are inverse proportional to line profile broadening. Because the parameter $b$ is proportional to crystallite size and profile broadening is inverse proportional to the crystallite size. And the parameter $d$ is inverse proportional to dislocation density and line profile broadening is proportional to the dislocation density. The tendencies of the WSSRs contour map among three graphs are similar; however, the depths and positions of valleys differ among the three plots. The value of WSSR was smallest at $b \approx 4$ and $d$ $\approx 100$ with $e=0.001$ in the three maps. Thus, the optimum values are reasonably expected to coincide with these values.
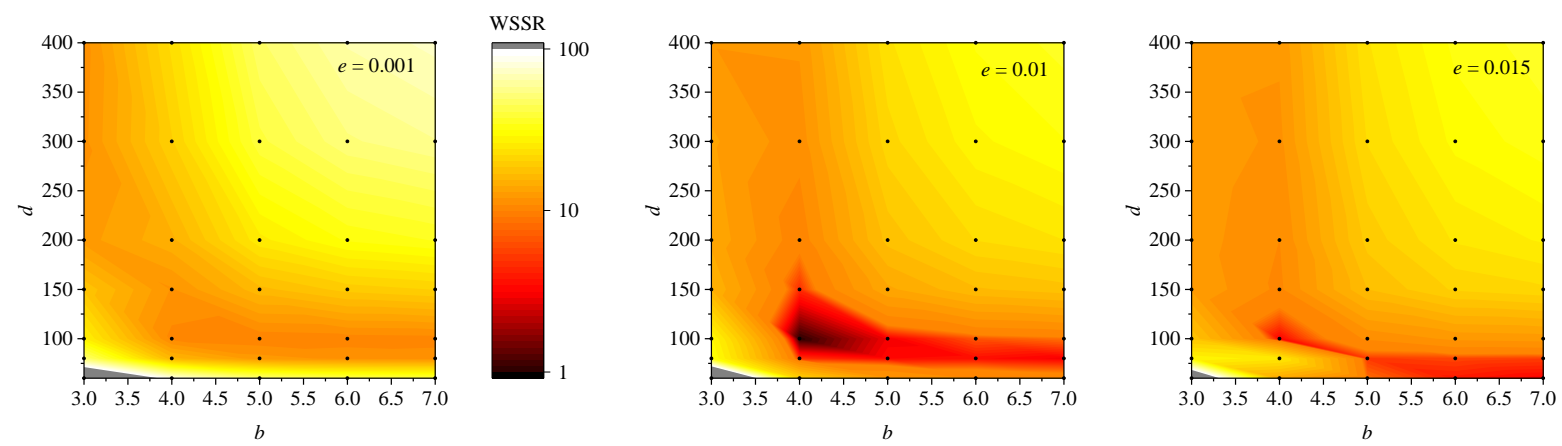

Fig. 2 WSSR contour maps at each value of $b$ and $d$; parameter $e$ was fixed at a) 0.001, b) 0.01 , and c) 0.015. The plots indicate the calculated conditions for $b$ and $d$. Colors are interpolated from the plots.
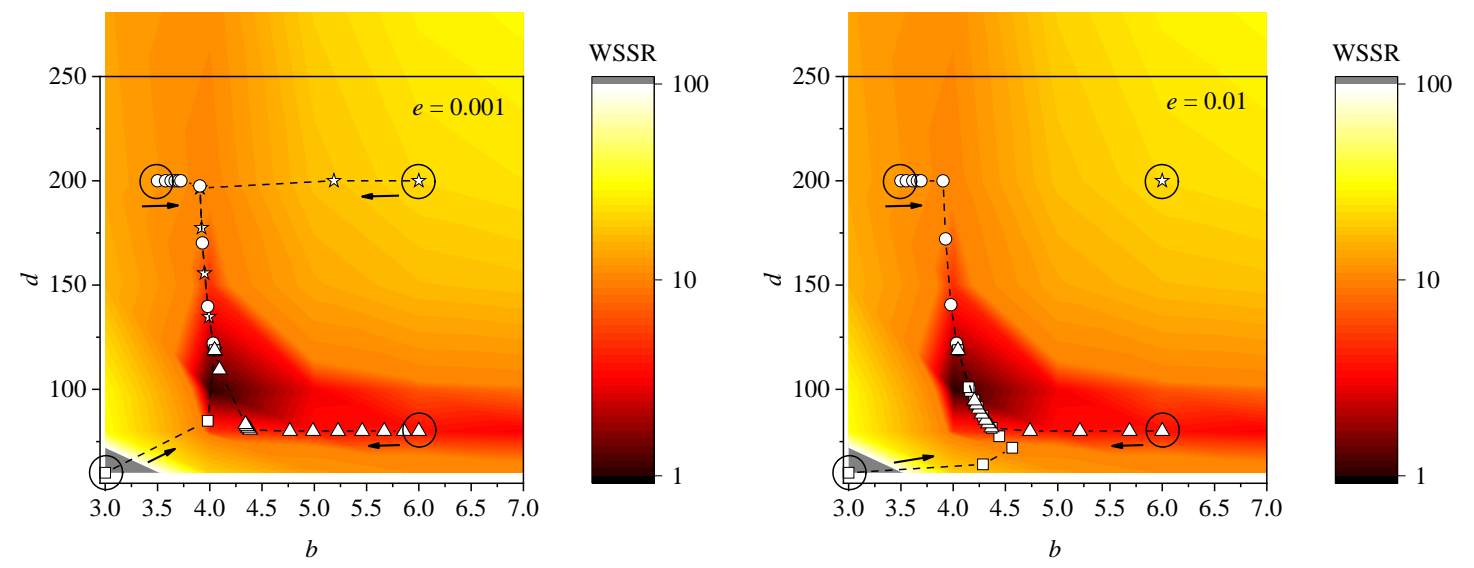

Fig. 3 Trajectories of values $b$ and $d$ as parameters $b, d$, and e are varied. Initial values of $e$ were 0.0001 and $0.01, b$ and $d$ were started from cicled points. The contour maps of the backgrounds are from Fig. $2 b$.

\section{In the case of parameters $b, d$, and $e$ as variables}

Fig. 3 shows the trajectories of the values of $b$ and $d$ in the WSSR contour maps (the contour map in Fig. 2b was used as the background) when $b, d$, and $e$ were variable and four initial values for $b$ and $d$ were arbitrarily chosen. Plots and lines on the maps show the trajectories of the $b$ and $d$ values during iterative calculations. When parameter $e$ was larger for a calculation with initial values $b=6$ and $d=200$, the iterative calculations stopped after 36 times and the values of the parameters did not change. On the contrary, when the initial value of $e$ was smaller ( $e=0.001$ ), parameters $b$ and $d$ changed with every the initial values. This tendency was also observed for the other values of $e$. These results suggest that the value of $e$ should be set smaller ( $e \leq 0.001)$ although the value $R_{\mathrm{e}}{ }^{*}=389 \mathrm{~nm}$ obtained from eq. (4) is too large in reality. 


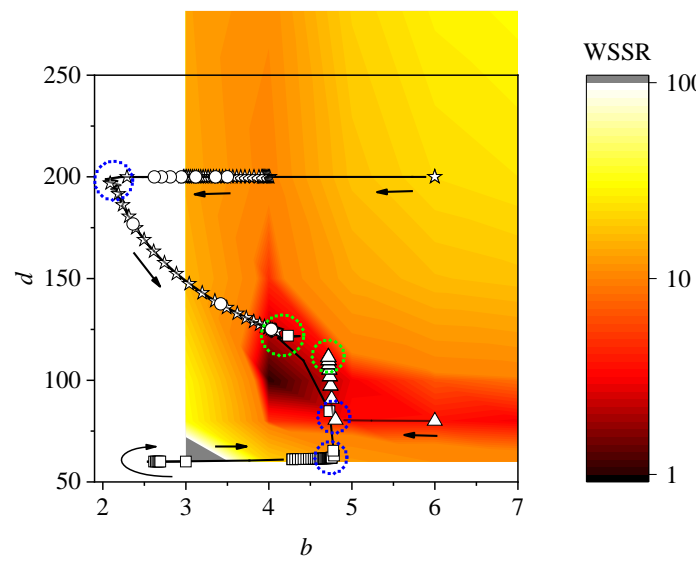

Fig. 4 Trajectories of the values $b$ and $d$ when all parameters were variable. Plots

are shown every 5 iterations. Green circles indicate converged positions. The contour maps of the backgrounds are from Fiq. $2 b$.
Murasawa et al. indicated that small values of $b$ and $d$ should be chosen [7]. In that study, $e$ was set to $0.1\left(R_{\mathrm{e}}{ }^{*}=38.9 \mathrm{~nm}\right)$ because the value is reasonable as effective radius for a dislocation. Therefore, small values of the parameters $b$ and $d$ were appropriate in that study.

\section{In the case of all parameters as variables}

Fig. 4 shows the trajectories of the $b$ and $d$ values in optimizations with four initial values for $b$ and $d$ when all parameters were variable. When the initial values of $b$ and $d$ were large (marked as is in Fig. 4), parameter $b$ varied toward smaller values, whereas $d$ did not vary. When $b$ reached approximately 2, parameters $b$ and $d$ simultaneously varied toward their optimum values. When initial values of $b=3.5$ and $d=200$ (marked as $\bigcirc$ ) were used, the path was almost the same as the case $b=$ 3.5 and $d=200$. When both parameters $b$ and $d$ were small (marked as $\square$ ), parameter $b$ initially varied toward smaller values and then increased, whereas parameter $d$ varied little. Parameter $d$ then varied toward larger values as parameter $b$ varied. When $b=6$ and $d=60$ (marked as $\triangle$ ), parameter $b$ varied toward smaller values and stopped at $4.5 \leq b \leq 5$, which corresponds to a position on the trace where $b$ and $d$ were small. Then, only parameter $d$ varied toward larger values. The optimized values of $b$ and $d$ differed substantially from the other initial conditions.

When all parameters were variable, the converged values reached from a given initial condition differed when the initial condition was changed. However, in all cases, the parameters almost reached their assumed optimum values. In a case where only three parameters were variable, calculations stopped in the early steps of iteration without variation of the parameter values; this termination was likely due to a low degree of freedom. The termination of iterations is controlled by the difference in WSSRs before and after each calculation ( $\triangle$ WSSRs). Even if the slope of contour of the WSSRs due to the values of $b$ and $d$ is small, the other parameters must change to avoid exceeding the limit of $\Delta$ WSSRs; in this case, the parameter $e$ is the only variable parameter other than $b$ and $d$.

\section{Discussion}

The converged values are summarized in Table 2. All of the WSSRs were similar to 0.111 and sufficiently small. In the case of initial conditions $b=6$ and $d=80(\triangle)$, the parameters converged to different values than those reached under other initial conditions, as shown Fig. 4. Not only parameters $b$ and $d$ but also the other parameters, $a, c$, and $e$, were different.

Figure 5 shows typical microstructural characteristics calculated from the CMWP parameters using Eqs. (1)-(4). Although the differences are not fatal, the values of all of the parameters differed substantially, especially in the case of the aforementioned initial conditions. However, avoiding such initial conditions is better from the viewpoint of obtaining precise optimum values. When three parameters were variable, the parameters converged and agreed, independent of the initial values of $b$ and $d$ except in the case of a large initial value $e$. Therefore, before carrying out optimizations with all parameters variable, a pre-calculation should be performed with small values of $e$ and arbitrary values of $b$ and $d$. Then, a full calculation should be performed with all parameters variable using the three converged values obtained via the pre- 
calculation. In actuality, a full calculation was conducted with all parameters variable using the averaged converged values obtained from the calculation in which only three parameters were variable (Table 2). We thus obtained the following optimum values: $a=1.57, b=4.10, c=$ $0.688, d=124$, and $e=5.43 \times 10^{-3}$; the WSSR was 0.111 . These values agree with the other results in Table 2 with the exception of the unsuitable initial condition. In the procedure, roughly optimum values are obtained by pre-calculation and then precise solutions are obtained by full calculation.

Table 2 Optimized results obtained with several initial values of $b$ and $d$ in the analyses with all parameters or only three parameters variable.

\begin{tabular}{ccc|cccccc}
\hline \multicolumn{7}{c|}{ Initial condition } & \multicolumn{6}{c}{ Converged value } \\
\hline$b$ & $d$ & Symbol & $a$ & $b$ & $c$ & $d$ & $e \times 10^{-3}$ & WSSR \\
\hline \hline 6 & 200 & $\downarrow$ & 1.57 & 4.09 & 0.693 & 123.9 & 5.40 & 0.111 \\
\hline 3.5 & 200 & $\bigcirc$ & 1.57 & 4.10 & 0.692 & 123.9 & 5.40 & 0.111 \\
\hline 3 & 60 & $\square$ & 1.58 & 4.23 & 0.611 & 121.9 & 5.93 & 0.111 \\
\hline 6 & 80 & $\triangle$ & 1.70 & 4.71 & 0.046 & 112.6 & 8.11 & 0.112 \\
\hline $\begin{array}{l}\text { Average of results only } b, d, e \\
\text { varied }\end{array}$ & $\begin{array}{c}1.96 \\
\text { (fixed) }\end{array}$ & 4.05 & $\begin{array}{c}0.7 \\
\text { (fixed) }\end{array}$ & 118.8 & 4.49 & 0.114 \\
\hline
\end{tabular}
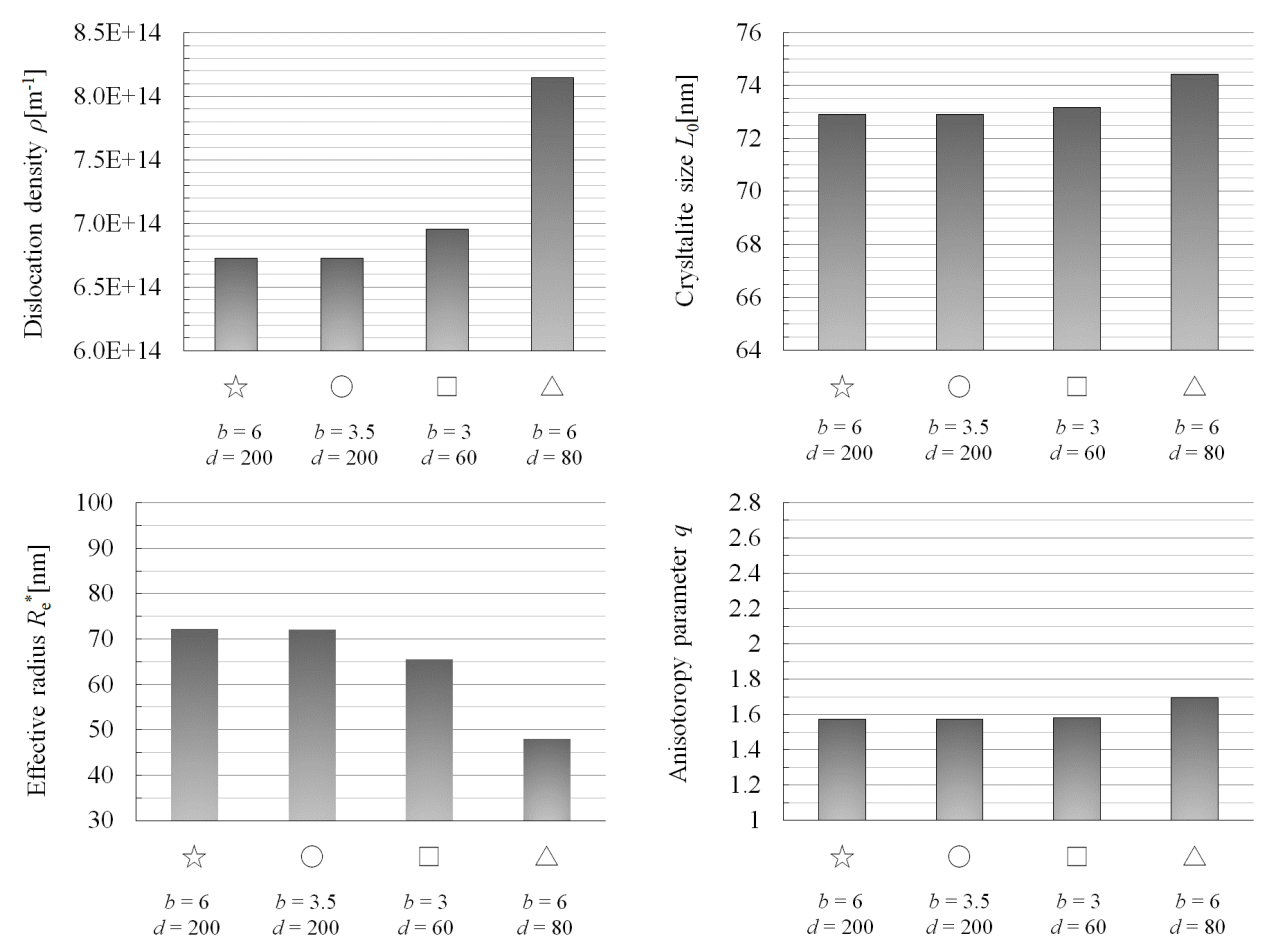

Fig. 5 Values of microstructural characteristics calculated from obtained CMWP parameters a-e in Table 2.

Fig. 6 shows the number of iterations in each calculation. The total iteration numbers of the pre- and full-calculations are approximately half the number of iterations of the calculations with all parameters simultaneously variable. An exception occurred when the initial values were $b=6$ and $d=80$, which were unsuitable initial values; in this case, both iteration numbers were the 


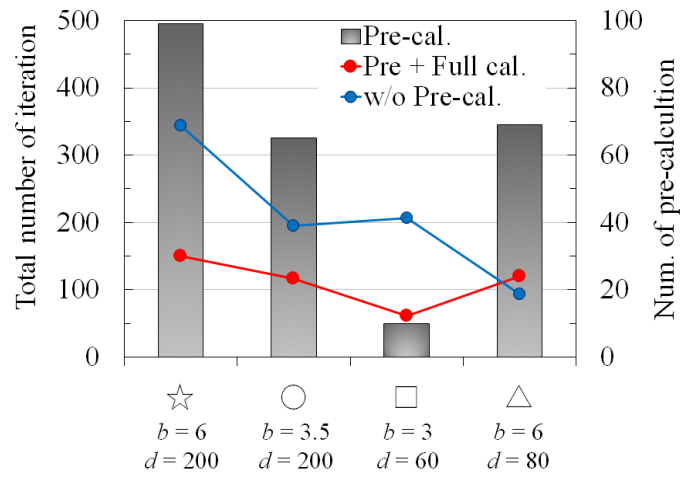

Fig. 6 Comparison of the number of iterations.

same. The two-step procedure is therefore effective in terms of both reliability of the converged values and time efficiency.

\section{Summary}

The convergence behavior in LPA using CMWP software was studied. Five parameters related to microstructural characteristics, $a-e$, were optimized using CMWP software. We observed the distributions of WSSRs on the space parameters $b$ and $d$ and obtained WSSR contour maps in $b-d$ space. We then showed the variation trajectory of parameters $b$ and $d$ under several initial sets of conditions where only the three parameters $b, d$, and $e$ were variable and where all parameters were variable. In the case where only three parameters were variable, the selection of a smaller value of $e$ tended to improve the stability of the calculations. In the case where all parameters were variable, although all of the results converged to similar values, they did not precisely agree. To obtain accurate optimum values, a two-step procedure is recommended. The procedure can lead to optimum solutions, with the additional advantage of time efficiency.

The neutron diffraction experiment was performed as a proposed program (2015A0294) at MLF, J-PARC. We thank Dr. Harjo, Dr. Kawasaki, Ms. Saori Iwata, and Mr. Hayato Komine for their experimental assistance.

\section{References}

[1] E. J. Mittemeijer and U. Welzel, in Mod. Diffr. Methods, Wiley-VCH (2013), pp. 87-126. https://doi.org/10.1002/9783527649884.ch4

[2] T. Ungár and A. Borbély, Appl. Phys. Lett. 69, 3173 (1996).

[3] P. Scardi, M. Leoni, and Y. H. Dong, Eur. Phys. J. B 18, 23 (2000). https://doi.org/10.1007/s100510070073

[4] G. Ribárik, T. Ungár, and J. Gubicza, J. Appl. Crystallogr. 34, 669 (2001). https://doi.org/10.1107/S0021889801011451

[5] P. Scardi and M. Leoni, Acta Crystallogr. Sect. A Found. Crystallogr. 58, 190 (2002). https://doi.org/10.1107/S0108767301021298

[6] G. Ribárik, Modeling of Diffraction Patterns Based on Microstructural Properties, Eötvös Loránd University, 2008.

[7] K. Murasawa, M. Takamura, M. Kumagai, Y. Ikeda, H. Suzuki, Y. Otake, T. Hama, and S. Suzuki, Mater. Trans. 59, 1135 (2018). https://doi.org/10.2320/matertrans.M2017380 